\title{
Computation of Hilbert Transform via Discrete Cosine Transform
}

\author{
H. Olkkonen ${ }^{1}$, P. Pesola ${ }^{2}$, J. T. Olkkonen ${ }^{3}$
}

${ }^{1}$ Department of Physics and Mathematics, University of Eastern Finland, Kuopio, Finland; ${ }^{2}$ Cognitive Neurobiology Laboratory, A.I.V. Institute of Molecular Sciences, University of Eastern Finland, Kuopio, Finland; ${ }^{3}$ VTT Technical Research Centre of Finland, Espoo, Finland.

Email: hannu.olkkonen@uef.fi

Received August 20 $0^{\text {th }}, 2010$; revised September 14 $4^{\text {th }}, 2010$; accepted September $19^{\text {th }}, 2010$.

\begin{abstract}
Hilbert transform (HT) is an important tool in constructing analytic signals for various purposes, such as envelope and instantaneous frequency analysis, amplitude modulation, shift invariant wavelet analysis and Hilbert-Huang decomposition. In this work we introduce a method for computation of HT based on the discrete cosine transform (DCT). We show that the Hilbert transformed signal can be obtained by replacing the cosine kernel in inverse DCT by the sine kernel. We describe a FFT-based method for the computation of HT and the analytic signal. We show the usefulness of the proposed method in mechanical vibration and ultrasonic echo and transmission measurements.
\end{abstract}

Keywords: Hilbert Transform, Analytic Signal, Envelope Analysis, FFT

\section{Introduction}

Hilbert transform (HT) plays an essential role in constructing analytic signals for a variety of signal and image processing applications. Conventionally, the HT has been used in envelope and instantaneous frequency analysis and as a core part in amplitude demodulators. The recent HT applications include Hilbert-Huang decomposition [1] and the computation of the shift invariant wavelet transform [2-5]. The applications of HT extend from geophysical [6], seismic, ultrasonic and radar to biomedical signals [7-10] and speech recognition systems [11]. The theoretical basis of HT is well established, but the computational procedures are still being developed. The most frequently used methods are based on the fast Fourier transform (FFT) [12], but many other methods have been proposed, such as digital filtering [12,13], the parametric modelling approach $[14,15]$ and the discrete Hartley transform [16].

In this work we introduce a new method for computation of HT, which is based on the discrete cosine transform (DCT) [17-21]. The DCT has become the industry standard in signal processing society (digital filtering, data compression, image coding, HDTV etc.). The properties of the DCT are very close to the statistically optimal Karhunen-Loeve transform (KLT) for a large number of signal families. The KLT decomposes the signal into uncorrelated signal vectors and minimizes the mean square error between a truncated representation and the actual signal. First, we consider the properties of HT and the related analytic signal. Then we review the FFT-based method for computation of the analytic signal. Finally, we introduce the DCT based method for computation of HT and describe some experimental results.

\section{Theoretical Considerations}

\subsection{Hilbert Transform}

Hilbert transform has been frequently used to obtain an analytic signal defined as

$$
x_{a}[n]=x[n]+j \overline{x[n]}
$$

where $x[n]$ denotes the Hilbert transform of the discrete time signal $x[n], \mathrm{n}=0,1, \cdots, \mathrm{N}-1$ and $j=\sqrt{-1}$. The discrete time signal $\mathrm{x}[\mathrm{n}]$ is obtained by sampling the continuous time signal at $\mathrm{T}$ intervals. In this work we use the normalization $\mathrm{T}=1$.

The Fourier transform of the analytic signal has the following property

$$
X_{a}(j \omega)=\left\{\begin{array}{cc}
2 X(j \omega) & 0 \leq \omega<\pi \\
0 & -\pi \leq \omega<0 .
\end{array}\right.
$$

On the other hand, Fourier transform of $\overline{x[n]}$ is of the form 


$$
\overline{X(j \omega)}= \begin{cases}-j X(j \omega) & 0 \leq \omega<\pi \\ j X(j \omega) & -\pi \leq \omega<0 .\end{cases}
$$

A common use of the Hilbert transform is to recover the amplitude information of the modulated sinusoidal waveforms. As an example let us consider a complex sinusoidal signal

$$
x[n]=a[n] e^{-j(\omega n+\phi)} .
$$

The time dependent amplitude $a[n]$ may be reconstructed from

$$
A[n]=\left|x_{a}[n]\right|=\sqrt{x[n]^{2}+\overline{x[n]}^{2}} .
$$

\subsection{FFT Based Computation of the Analytic Signal}

The discrete Fourier transform (DFT) of the signal $x[n]$, $\mathrm{n}=0,1, \cdots, \mathrm{N}-1$ is defined as

$$
X[k]=\sum_{n=0}^{N-1} x[n] W_{N}^{k n} \quad k=0, \cdots, N-1,
$$

where $W_{N}^{k n}=\mathrm{e}^{-2 \pi j k n / N}$. The inverse transform (IDFT) is defined as

$$
x[n]=\frac{1}{N} \sum_{k=0}^{N-1} X[k] W_{N}^{-k n} \quad n=0, \cdots, N-1 .
$$

The computational complexity of the DFT via the FFT is only $\mathrm{O}(\mathrm{N} \log \mathrm{N})$ for even $\mathrm{N}$. We use a short notation $X[k]=F F T_{N}\{x[n]\}$ for FFT of the signal $x[n]$. The computation of analytic signal using the FFT- based method is based on the property (2) of the Fourier spectrum of the analytic signal. If $\mathrm{X}[\mathrm{k}](\mathrm{k}=0, \cdots, \mathrm{N}-1)$ denotes the DFT coefficients of the original signal, the $X[k]$ $(\mathrm{k}=\mathrm{N} / 2, \cdots, \mathrm{N}-1)$ represent the values in the negative frequency band $\quad(-\pi \leq \omega<0)$. By zeroing those coefficients the inverse FFT yields the analytic signal. A more precise result is obtained by weighting the DFT coefficients by a windowing function given by [12]

$$
w[k]=\left\{\begin{array}{cc}
2 & k=1,2, \cdots, N / 2-1 \\
1 & k=0 \text { and } N / 2 \\
0 & k=N / 2+1, \cdots, N-1 .
\end{array}\right.
$$

The analytic signal is then computed from

$$
x_{a}[n]=\operatorname{IFFT}_{N}\{w[k] X[k]\} .
$$

The weighting sequence based algorithm (9) is used in the Matlab built-in routine hilbert.m for computation of the analytic signal.

\subsection{The Discrete Cosine Transform}

For the real-valued data sequence $x[n], \mathrm{n}=0,1, \ldots, \mathrm{N}-1$ the DCT [16] is defined as

$$
\begin{aligned}
& Y[k]=a_{k} \sum_{n=0}^{N-1} x[n] \cos [\pi k(2 n+1) / 2 N], \\
& k=0,1, \cdots, N-1,
\end{aligned}
$$

and the corresponding inverse DCT as

$$
x[n]=\sum_{k=0}^{N-1} a_{k} Y[k] \cos [\pi k(2 n+1) / 2 N],
$$

where the normalization constant $a_{0}=\sqrt{1 / N}$ for $\mathrm{k}=0$, and $a_{k}=\sqrt{2 / N}$ for $\mathrm{k}=1,2, \cdots, \mathrm{N}-1$.

The fast algorithms for computation of DCT are based on the FFT [12] or they are based on the direct factorization of the DCT matrix [17].

\section{Computation of Hilbert Transform}

\subsection{Computation of HT Via the DCT}

The key idea of the present work is to write the IDCT kernel in (11) as

$$
\begin{aligned}
K(n, k) & =\cos [\pi k(2 n+1) / 2 N] \\
& =\cos (\pi k / 2 N) \cos (\pi k n / N) \\
& -\sin (\pi k / 2 N) \sin (\pi k n / N) .
\end{aligned}
$$

We may consider the variable $\mathrm{k}$ in (11) is related to the discrete frequency $\omega_{k}=2 \pi k / N \quad(k=0,1, \cdots, \mathrm{N}-1)$. Then we obtain

$$
\begin{aligned}
K(n, k) & =\cos \left(\omega_{k} / 4\right) \cos \left(\omega_{k} n / 2\right) \\
& -\sin \left(\omega_{k} / 4\right) \sin \left(\omega_{k} n / 2\right) .
\end{aligned}
$$

In Appendix I we show that the Hilbert transforms of the cosine and sine functions are

$$
\begin{aligned}
& \overline{A \cos (\omega n)}=\left\{\begin{array}{rll}
A \sin (\omega n) & \text { when } & 0 \leq \omega<\pi \\
-A \sin (\omega n) & \text { when } & -\pi \leq \omega<0
\end{array}\right. \\
& \overline{A \sin (\omega n)}=\left\{\begin{array}{l}
-A \cos (\omega n) \text { when } 0 \leq \omega<\pi \\
A \cos (\omega n) \text { when }-\pi \leq \omega<0 .
\end{array}\right.
\end{aligned}
$$

When $\mathrm{k}$ varies from 0 to $N-1$, the frequency $\omega=\omega_{k} / 2$ in (13) varies between $0 \leq \omega<\pi$. Applying it to $(11,12)$ we receive the Hilbert transform of the IDFT kernel as

$$
\begin{aligned}
\overline{K(n, k)} & =\cos \left(\omega_{k} / 4\right) \sin \left(\omega_{k} n / 2\right) \\
& +\sin \left(\omega_{k} / 4\right) \cos \left(\omega_{k} n / 2\right) \\
& =\sin [\pi k(2 n+1) / 2 N] .
\end{aligned}
$$

Finally, we obtain The Hilbert transform of the discrete time signal (11) as 
$\overline{x[n]}=\sum_{k=0}^{N-1} a_{k} Y[k] \overline{K(n, k)}=\sum_{k=0}^{N-1} a_{k} Y[k] \sin [\pi k(2 n+1) / 2 N]$.

\subsection{FFT Based Computation of HT from the DCT Coefficients}

An interesting relation is obtained from $(11,16)$ for the computation of the analytic signal as

$$
\begin{aligned}
x_{a}[n] & =x[n]+j \overline{x[n]} \\
& =\sum_{k=0}^{N-1} a_{k} Y[k]\{\cos [\pi k(2 n+1) / 2 N] \\
& +j \sin [\pi k(2 n+1) / 2 N]\},
\end{aligned}
$$

which yields

$$
x_{a}[n]=\sum_{k=0}^{N-1} c_{k} Y[k] e^{j \pi k n / N},
$$

where $c_{k}=a_{k} e^{j \pi k / 2 N}$. We get the even sequence of the analytic signal as

$$
\begin{aligned}
x_{a}[2 n] & =\sum_{k=0}^{N-1} c_{k} Y[k] e^{j 2 \pi k n / N}=N \operatorname{IFFT}_{N}\left\{c_{k} Y[k]\right\}, \\
n & =0,1, \cdots, N / 2-1
\end{aligned}
$$

and the odd sequence as

$x_{a}[2 n+1]=\sum_{k=0}^{N-1} d_{k} Y[k] e^{j 2 \pi k n / N}=N \operatorname{IFFT}_{N}\left\{d_{k} Y[k]\right\}$.

where $d_{k}=c_{k} e^{j \pi k / N}$. However, the odd sequence can be computed from the following symmetry relation

$x_{a}[2 n+1]=x_{a}^{*}[2(N-1-n)], n=0,1, \cdots, N / 2-1$,

where $(*)$ denotes complex conjugate. The equation (21) can be proved by substituting $N-1-n$ for $n$ in (19). Consequently, even values of the analytic signal $x_{a}[2 n]$ for $\mathrm{n}=0,1,2, \cdots, \mathrm{N} / 2-1$ can be computed from the DCT coefficients via one N-point IFFT (18) and the odd sequence $x_{a}[2 n+1]$ from the symmetry relation (21). It is also possible to split (19) and (20) into two (N/2)- point IFFTs (see Appendix II).

\section{Experimental Results}

The present algorithm was tested for various signals, whose HT could be computed analytically from the convolution integral (Appendix I). There was a good agreement with the analytically computed HT and the results obtained by the DCT-based algorithm. As an example, Figure 1 shows the HT of the signal $x(t)=\sin (0.10 t)+\cos (0.12 t)$, where the mean error between the computed and analytically computed HT $\overline{x(t)}=-\cos (0.10 t)+\sin (0.12 t)$ was $3 \times 10^{-5}$.
The usefulness of the DCT-based algorithm was tested in envelope analysis of different experimental signals. Figure 2 shows the vibration measurement of the running $\mathrm{AC}$ motor using an acceleration sensor. The signal is relatively highly noised and the computed envelope is disturbed by the noise components. Using only $1 / 4$ of the DCT coefficients the smoothed version of the envelope is obtained. A typical example is the measurement of the ultrasonic echo signal from different materials. Figure 3 shows the echo from the relatively diffuse homogenous material, $30 \mathrm{~cm}$ thick cellulose layer, moisture content (MC $3 \%$ ). The exponential tail (envelope) computed by the present method follows close to the amplitude of the measured curve. Figure 4 shows an ultrasonic echo from material, which consists of two layers of diffuse material, $5 \mathrm{~cm}$ cellulose (MC $3 \%$ ) and $25 \mathrm{~cm}$ cellulose (MC $9 \%$ ). Also in this case the envelope follows precisely the amplitude distribution.

\section{Discussion and Conclusions}

The DCT coefficients $Y[k](10)$ are high for the low values of $k$ and without notable error the rest of the coefficients can be zeroed. If the signal is buried by noise, the truncation of the DCT coefficients reduces noise in the computed HT and the envelope (Figure 2). In the FFT-based method (9), in Hartley transform assisted method [16] and in other algorithms described in literature [14-15] this usually cannot be made. In this context the DCT-based method is more robust to noise.

The DCT-based computation of HT requires the computation of the DCT coefficients, which needs about half of the multiplications compared with the FFT implementation using e.g., the DCT algorithm

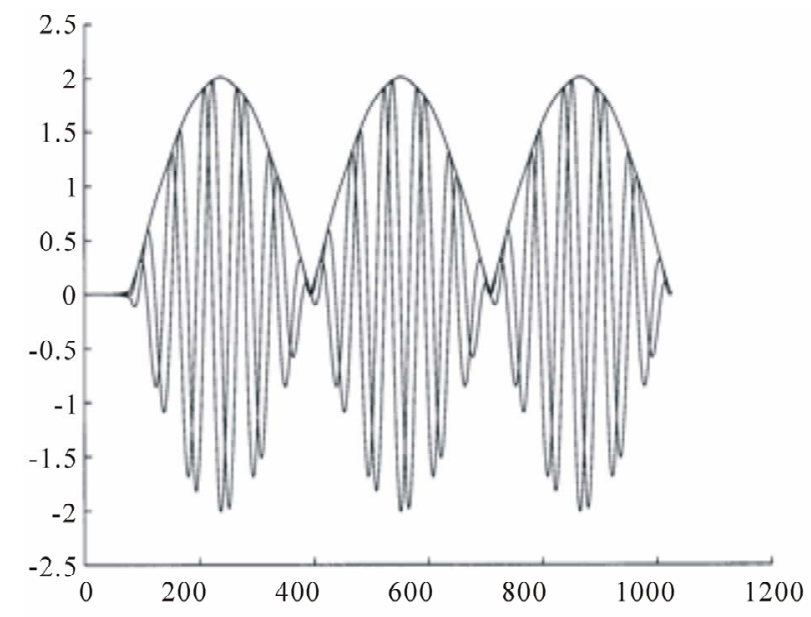

Figure 1. The envelope (slowly varying amplitude) and the real and imaginary parts of the Hilbert transform of the signal $x(t)=\sin (0.10 t)+\cos (0.12 t)$. 
based on the discrete Hartley transform [21]. The computation of the HT from the DCT coefficients given by (17-19) requires computation of one $\mathrm{N}$-point IFFT. Hence, the proposed algorithm needs about $3 / 4$ multiplications compared with the FFT-based approach (9). An alternative algorithm (Appendix II) requires two (N/2)-point IFFTs, which makes it slightly faster compared with the N-point IFFT. However, in hardware implementation the speed can be doubled by using two parallel (N/2)-point IFFT chips for computation of (31).

The distinct difference between the proposed method and the FFT-based method is in the Fourier spectrum of the computed analytic signal. Due to window weighting given by $(8,9)$, the negative frequency components in the FFT-based method are zero. In the
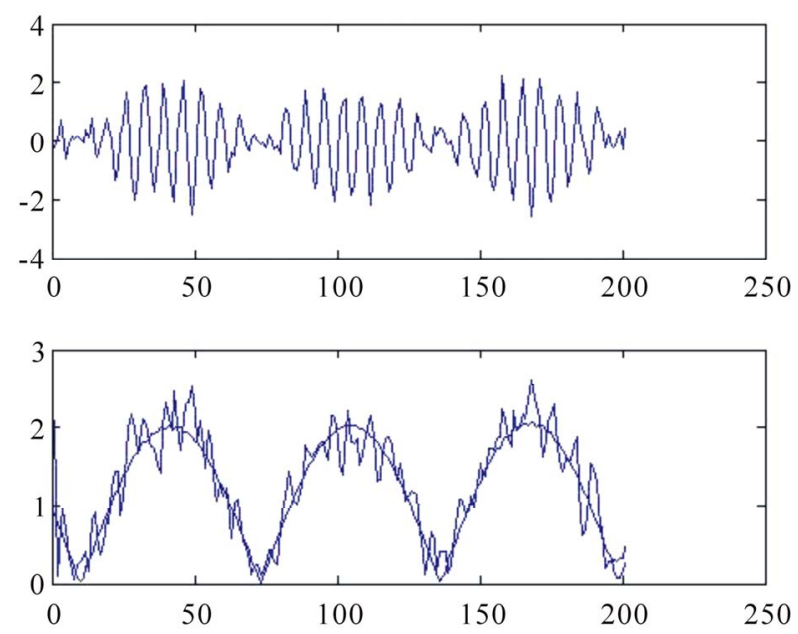

Figure 2. The measurement of the vibration of the AC motor using the acceleration sensor (top). The envelope of the signal and the smoothed envelope using only 1/4 of the DCT coefficients (bottom).

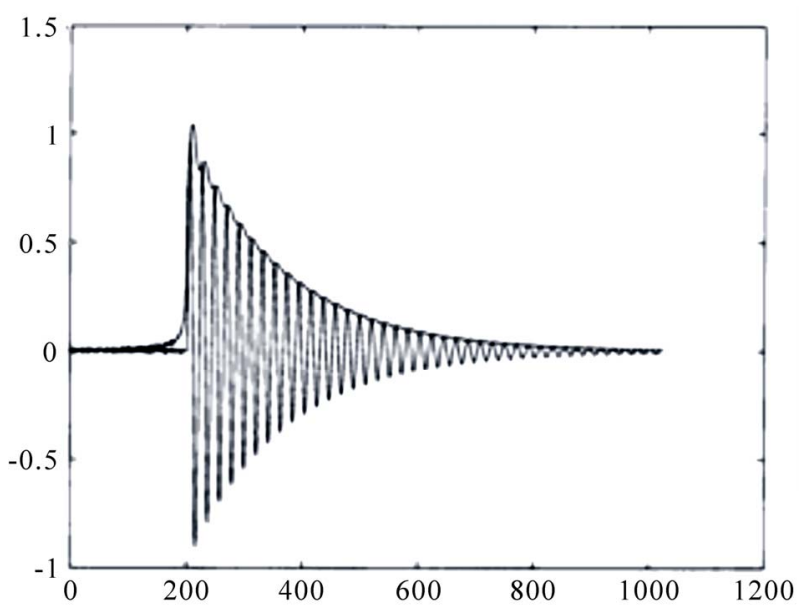

Figure 3. Envelope analysis of the ultrasonic echo from the homogenous material.

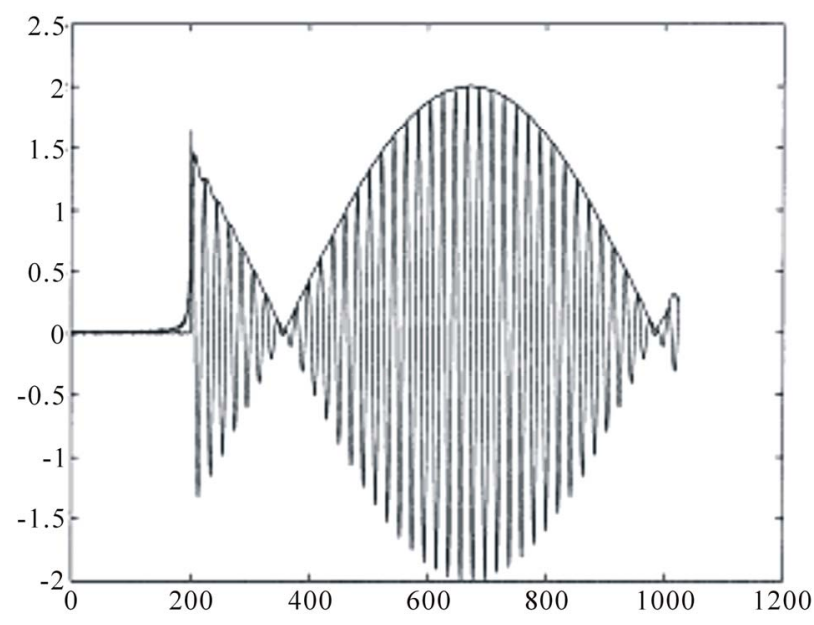

Figure 4. Envelope analysis of the ultrasonic echo from two layers of homogenous material with different moisture contents.

present algorithm the negative frequency components are very small (typically of the order of $10^{-4}$ ), but not precisely zero. The reason is that our approach is based on the convolution property (22) (see Appendix I), and not on the properties of the Fourier spectrum of the analytic signal (2).

The usefulness of the present method was tested in connection with the ultrasonic echo measurements. The primary aim in our experiments was to develop a method for non-destructive characterization of the insulating materials (thickness, humidity distribution etc.). The Hilbert transformed echo signal seems highly promising in this context (Figures 3,4).

In conclusion, this work proposes a new DCT-based method for computation of HT and the analytical signal. Preliminary experimental studies showed that the method is faster and more robust to noise than the FFT-based method. The present method can be easily extended to the computation of HT of 2D signals via 2D DCT algorithm.

\section{Acknowledgements}

The authors are indebted to the reviewer's comments, which improved the manuscript significantly.

\section{REFERENCES}

[1] N. E. Huang, Z. Shen, and S. R. Long, M. C. Wu, E. H. Shih, Q. Zheng, C. C. Tung, and H. H. Liu, "The Empirical Mode Decomposition and the Hilbert Spectrum for Nonlinear and Nonstationary Time Series Analysis," Proceedings of the Royal Society of London A, Vol. 454, pp. 903-995, 1998.

[2] I. W. Selesnick, "Hilbert Transform Pairs of Wavelet Bases,” IEEE Signal Processing Letters, Vol. 8, No. 6, 
June 2001, pp. 170-173.

[3] H. Ozkaramanli and R. Yu, "On the Phase Condition and Its Solution for Hilbert Transform Pairs of Wavelet Bases,” IEEE Transactions on Signal Processing, Vol. 51, No. 12, December 2003, pp. 3293-3294.

[4] R. Yu and H. Ozkaramanli, "Hilbert Transform Pairs of Biorthogonal Wavelet Bases," IEEE Transactions on Signal Processing, Vol. 54, No. 6, part 1, June 2006, pp. 2119-2125.

[5] H. Olkkonen, J. T. Olkkonen and P. Pesola, "FFT Based Computation of Shift Invariant Analytic Wavelet Transform,” IEEE Signal Processing Letters, Vol. 14, No. 3, March 2007, pp. 177-180.

[6] W. M. Moon, A. Ushah and B. Bruce, "Application of 2-D Hilbert Transform in Geophysical Imaging with Potential Field Data," IEEE Transactions on Geoscience and Remote Sensing, Vol. 26, No. 5, September 1988, pp. 502-510.

[7] H. Olkkonen, P. Pesola, J. T. Olkkonen and H. Zhou, "Hilbert Transform Assisted Complex Wavelet Transform for Neuroelectric Signal Analysis," Journal of Neuroscience Methods, Vol. 151, No. 2, pp. 106-113, 2006.

[8] H. Kanai, Y. Koiwa and J. Zhang, "Real-Time Measurements of Local Myocardium Motion and Arterial Wall Thickening," IEEE Transactions on Ultrasonics, Ferroelectrics, and Frequency Control, Vol. 46, No. 5, September 1999, pp. 1229-1241.

[9] S. M. Shors, A. V. Sahakian, H. J. Sih and S. Swiryn, “A Method for Determining High-Resolution Activation Time Delays in Unipolar Cardiac Mapping,” IEEE Transactions on Biomedical Engineering, Vol. 43, No. 12, December 1996, pp. 1192-1196.

[10] A. K. Barros and N. Ohnishi, "Heart Instantaneous Frequency (HIF): An Alternative Approach to Extract Heart Rate Variability,” IEEE Transactions on Biomedical Engineering, Vol. 48, No. 8, August 2001, pp. 850-855.

[11] O. W. Kwon and T. W. Lee, "Phoneme Recognition Us- ing ICA-Based Feature Extraction and Transformation," Signal Processing, Vol. 84, No. 6, 2004, pp. 1005-1019.

[12] A. V. Oppenheim and R. W. Schafer, "Discrete-Time Signal Processing," Prentice-Hall, Englewood Cliffs, 1989.

[13] K. L. Peacock, "Kaiser-Bessel Weighting of the Hilbert Transform High-Cut Filter," IEEE Transactions on Acoustics, Speech, and Signal Analysis, Vol. 33, No. 1, February 1985, pp. 329-331.

[14] A. Rao and R. Kumaresan, "A Parametric Modeling Approach to Hilbert Transformation,” IEEE Signal Processing Letters, Vol. 5, No. 1, January 1998, pp. 15-17.

[15] R. Kumaresan, "An Inverse Signal Approach to Computing the Envelope of a Real Valued Signal,” IEEE Signal Processing Letters, Vol. 5, No. 10, October 1998, pp. 256-259.

[16] S. C. Pei and S. B. Jaw, "Computation of Discrete Hilbert Transform through Fast Hartley Transform,” IEEE Transactions on Circuits and Systems, Vol. 36, No. 9, 1989, pp. 1251-1252.

[17] N. Ahmed, T. Natajaran and K. R. Rao, "Discrete Cosine Transform,” IEEE Transactions on Computers, Vol. 23, 1974, pp. 90-94.

[18] C. W. Kok, "Fast Algorithm for Computing Discrete Cosine Transform," IEEE Transactions on Signal Processing, Vol. 45, No. 3, March 1997, pp. 757-760.

[19] G. Bi and L. W. Yu, "DCT Algorithms for Composite Sequence Lengths,” IEEE Transactions on Signal Processing, Vol. 46, No. 3, March 1998, pp. 554-562.

[20] V. Britanak, P. Yip and K. R. Rao, Discrete Cosine and Sine Transforms: General Properties, Fast Algorithms and Integer Approximations, Academic Press Inc., Elsevier Science, Amsterdam, 2007

[21] H. Malvar, "Fast Computation of the Discrete Cosine Transform and the Discrete Hartley Transform,” IEEE Transactions on Acoustics, Speech, Signal Processing, Vol. 35, No. 10, October 1987, pp. 1484-1485. 


\section{Appendices}

\section{Hilbert Transform of the Cosine Function}

The Hilbert transform of the continuous time signal $\mathrm{x}(\mathrm{t})$ can be defined by the convolution as

$$
\overline{x(t)}=\frac{1}{\pi t} * x(t)=\frac{1}{\pi} \int_{-\infty}^{\infty} \frac{x(\tau)}{n-\tau} d \tau, \quad n=0,1, \cdots, N-1 .
$$

For the discrete time signal $\mathrm{x}[\mathrm{n}]$ we may write

$$
\overline{x[n]}=\frac{1}{\pi} \int_{-\infty}^{\infty} \frac{x(t)}{n-t} d t, \quad n=0,1, \cdots, N-1 .
$$

Now we may calculate the Hilbert transform of $x[n]=A \cos (\omega n)$

$$
\overline{A \cos (\omega n)}=\frac{A}{\pi} \int_{-\infty}^{\infty} \frac{\cos (\omega t)}{n-t} d t=\frac{A}{\pi} \int_{-\infty}^{\infty} \frac{\cos (\omega(n-t))}{t} d t .
$$

The last result is due to the general property of the convolution integral

$$
\int x(t-\tau) y(\tau) d \tau=\int x(\tau) y(t-\tau) d \tau .
$$

By expanding

$\cos (\omega(n-t))=\cos (\omega n) \cos (\omega t)+\sin (\omega n) \sin (\omega t) \quad$ we obtain

$$
\overline{A \cos (\omega n)}=\frac{A}{\pi} \cos (\omega n) \int_{-\infty}^{\infty} \frac{\cos (\omega t)}{t} d t+\frac{A}{\pi} \sin (\omega n) \int_{-\infty}^{\infty} \frac{\sin (\omega t)}{t} d t .
$$

Since we have

$$
\int_{-\infty}^{\infty} \frac{\cos (\omega t)}{t} d t=0, \quad \int_{-\infty}^{\infty} \frac{\sin (\omega t)}{t} d t=\pi
$$

we obtain the final result

$$
\overline{A \cos (\omega n)}=\left\{\begin{aligned}
A \sin (\omega n) & \text { when } \quad 0 \leq \omega<\pi \\
-A \sin (\omega n) & \text { when } \quad-\pi \leq \omega<0 .
\end{aligned}\right.
$$

In a similar manner we may prove

$$
\overline{A \sin (\omega n)}=\left\{\begin{array}{l}
-A \cos (\omega n) \text { when } \quad 0 \leq \omega<\pi \\
A \cos (\omega n) \text { when }-\pi \leq \omega<0 .
\end{array}\right.
$$

\section{Alternative Computational Method for (17)} and (18)

We may split (17) into two parts

$$
\begin{aligned}
x_{a}[2 n]= & \sum_{k=0}^{N-1} c_{k} Y[k] e^{j 2 \pi k n / N}=\sum_{k=0}^{N / 2-1} c_{2 k} Y[2 k] e^{j 2 \pi k n /(N / 2)} \\
& +c_{2 k+1} Y[2 k+1] e^{j 2 \pi n / N} e^{j 2 \pi k n /(N / 2)},
\end{aligned}
$$

which gives the even values

$$
\begin{aligned}
x_{a}[2 n]= & \frac{N}{2} \operatorname{IFFT}_{N / 2}\left\{c_{2 k} Y[2 k]\right\} \\
& +e^{j 2 \pi n / N} \frac{N}{2} \operatorname{IFFT}_{N / 2}\left\{c_{2 k+1} Y[2 k+1]\right\} .
\end{aligned}
$$

Correspondingly, the odd values are obtained from

$$
\begin{aligned}
x_{a}[2 n+1]= & \frac{N}{2} \operatorname{IFFT}_{N / 2}\left\{d_{2 k} Y[2 k]\right\}+ \\
& e^{j 2 \pi n / N} \frac{N}{2} \operatorname{IFFT}_{N / 2}\left\{d_{2 k+1} Y[2 k+1]\right\} .
\end{aligned}
$$

If we denote the even values in (28) as

$$
x_{a}[2 n]=x_{1}[n]+x_{2}[n],
$$

then the odd values are obtained from

$$
x_{a}[2 n+1]=x_{1}^{*}[N / 2-1-n]-x_{2}^{*}[N / 2-1-n] .
$$

The result (34) can be proved by direct substitution of $N / 2-1-n$ for $n$ in (30). 